\title{
A New Subclass of Analytic Functions Defined by Using Salagean $q$-Differential Operator
}

\author{
Muhammad Naeem $^{1}$, Saqib Hussain ${ }^{2, *}$, Tahir Mahmood ${ }^{1}\left(\mathbb{D}\right.$, Shahid Khan $^{3}$ \\ and Maslina Darus ${ }^{4}$ (D) \\ 1 Department of Mathematics and Statistics, International Islamic University, 44000 Islamabad, Pakistan; \\ naeem.phdma75@iiu.edu.pk (M.N.); tahirbakhat@iiu.edu.pk (T.M.) \\ 2 Department of Mathematics, COMSATS University Islamabad, Abbottabad Campus, \\ 22060 Abbottabad, Pakistan \\ 3 Department of Mathematics, Riphah International University, 44000 Islamabad, Pakistan; \\ shahidmath761@gmail.com \\ 4 School of Mathematical Sciences, Faculty of Science and Technology, Universiti Kebangsaan Malaysia, \\ 43600 Selangor, Malaysia; maslina@ukm.edu.my \\ * Correspondence: sqaibhussain@cuiatd.edu.pk
}

Received: 29 March 2019; Accepted: 13 May 2019; Published: 21 May 2019

\begin{abstract}
In our present investigation, we use the technique of convolution and quantum calculus to study the Salagean $q$-differential operator. By using this operator and the concept of the Janowski function, we define certain new classes of analytic functions. Some properties of these classes are discussed, and numerous sharp results such as coefficient estimates, distortion theorem, radii of star-likeness, convexity, close-to-convexity, extreme points, and integral mean inequalities of functions belonging to these classes are obtained and studied.
\end{abstract}

Keywords: analytic functions; subordination; Salagean $q$-differential operator

MSC: Primary 30C45; Secondary 30C50

\section{Introduction}

Let $E=\{z \in \mathbb{C}:|z|<1\}$ be the open unit disk and $\mathcal{A}$ be the class of all functions $f$ that are analytic in $E$ and normalized by $f(0)=0$ and $f^{\prime}(0)=1$. Thus, each $f \in \mathcal{A}$ has the Maclaurin's series expansion of the form:

$$
f(z)=z+\sum_{n=2}^{\infty} a_{n} z^{n}
$$

The class of all such functions that are in $\mathcal{A}$ and univalent in $E$ is denoted by $S$; see [1]. A domain $D$, which is the subset of a complex set, is star shaped about any point $z_{0}$ if any other point of $D$ joining with $z_{0}$ by line segment lies with in $D$, while the domain $D$ is said to be convex if and only if it is starlike with respect to each of its points. The subclasses of $S$, which are starlike and convex, are denoted by $\mathcal{S}^{*}$ and $\mathcal{C}$, respectively.

In 1936, Roberston [2] introduced the classes of starlike and convex functions of order $\alpha$. In 1991, Goodman [1,3] introduced the classes of uniformly-starlike and uniformly-convex functions, which were extensively studied by Ronning and independently by Ma and Minda [4,5]. In 1999, 
Kanas and Wisniowska $[6,7]$ introduced the classes of $k$-uniformly convex and $k$-uniformly starlike functions denoted by $k-\mathcal{U C} \mathcal{V}$ and $k-\mathcal{S T}$ respectively defined as:

$$
f \in k-\mathcal{U C V} \Longleftrightarrow z f^{\prime} \in k-\mathcal{S T} \Longleftrightarrow f \in A \text { and } \operatorname{Re}\left\{\frac{\left(z f^{\prime}(z)\right)^{\prime}}{f^{\prime}(z)}\right\}>\left|\frac{z f^{\prime \prime}(z)}{f^{\prime}(z)}\right|, z \in E, k \geq 0 .
$$

The class $k-\mathcal{U C V}$ was discussed earlier in [8], also with some extra restrictions and without geometrical interpretation by Bharati et al. [9]. Several authors investigated the properties of the subclasses of $\mathcal{S}^{*}$ and $\mathcal{C}$ with their generalizations in several directions; for a detailed study, see $[6,10-18]$.

If $f$ and $g$ are analytic in $E$, we say that $f$ is subordinate to $g$, written as $f \prec g$, if there exists a Schwarz function $w$, which is analytic in $E$ with $w(0)=0$ and $|w(z)|<1$ such that $f(z)=g(w(z))$. Furthermore, if the function $g$ is univalent in $E$, then we have the following equivalence:

$$
f(z) \prec g(z) \Longleftrightarrow f(0)=g(0) \text { and } f(E) \subset g(E) . \quad z \in E .
$$

For some detail, see $[1,19]$. Using the concept of subordination, Janowski introduced the class $P[A, B]$. A given analytic function $h$ with $h(0)=1$ is said to belong to the class $P[A, B]$, if and only if:

$$
h(z) \prec \frac{1+A z}{1+B z}, \quad-1 \leq B<A \leq 1 .
$$

Geometrically, a function $h(z) \in P[A, B]$ maps the open unit disk $E$ onto the domain $\Omega[A, B]$ defined by:

$$
\Omega[A, B]=\left\{w:\left|w-\frac{1-A B}{1-B^{2}}\right|<\frac{A-B}{1-B^{2}}\right\} .
$$

This domain represents an open circular disk centered on the real axis with diameter end points $D_{1}=\frac{1-A}{1-B}$ and $D_{2}=\frac{1+A}{1+B}$ with $0<D_{1}<1<D_{2}$.

Let $f, g \in \mathcal{A}$. Then, the convolution or Hadamard product of $f$ of the form (1) and $g$ of the form:

$$
g(z)=z+\sum_{n=2}^{\infty} b_{n} z^{n}
$$

is denoted by $f * g$ and defined as:

$$
(f * g)(z)=\sum_{n=0}^{\infty} a_{n} b_{n} z^{n} \quad(z \in E) .
$$

In the beginning of the last century, studies on $q$-difference equations appeared extensively especially by Carmichael [20], Jackson [21], Mason [22], and Trjitzinsky [23]. Research work in connection with function theory and $q$-theory together was first introduced by Ismail et al. [24]. Till now, only non-significant interest in this area has been shown, although it deserves more attention. Many differential and integral operators can be written in terms of convolution; for detail, we refer to [25-30]. It is worth mentioning that the technique of convolution helps researchers in further investigation of the geometric properties of analytic functions. For any non-negative integer $n$, the $q$-integer number $n$ denoted by $[n, q]$, is defined by:

$$
[n, q]=\frac{1-q^{n}}{1-q},[0, q]=0 .
$$

For non-negative integer $n$, the $q$-number shift factorial is defined by:

$$
[n, q] !=[1, q][2, q][3, q] \ldots[n, q], \quad([0, q] !=1) .
$$


We note that when $q \rightarrow 1,[n, q]$ ! reduces to the classical definition of factorial. Throughout this paper, we will assume $q$ to be a fixed number between zero and one. The $q$-difference operator related to the $q$-calculus was introduced by Andrews et al. [31]. For $f \in \mathcal{A}$, the $q$-derivative operator or $q$-difference operator is defined as:

$$
\mathcal{D}_{q} f(z)=\frac{f(q z)-f(z)}{z(q-1)}, \quad z \in E, q \neq 1
$$

It can easily be seen that for $n \in \mathbb{N}=\{1,2,3, \ldots\}$ and $z \in E$,

$$
\mathcal{D}_{q} z^{n}=[n, q] z^{n-1}, \quad \mathcal{D}_{q}\left\{\sum_{n=1}^{\infty} a_{n} z^{n}\right\}=\sum_{n=1}^{\infty}[n, q] a_{n} z^{n-1}
$$

Recently, Govindaraj and Sivasubramanian defined the Salagean $q$-differential operator [32] as: Let $f \in \mathcal{A}$. Then, the Salagean $q$-differential operator is defined as:

$$
\mathcal{D}_{q}^{0} f(z)=f(z), \quad \mathcal{D}_{q}^{1} f(z)=z \mathcal{D}_{q} f(z), \longrightarrow, \mathcal{D}_{q}^{i} f(z)=z \partial_{q}\left(\mathcal{D}_{q}^{i-1} f(z)\right)
$$

A simple calculation implies:

$$
\mathcal{D}_{q}^{i} f(z)=f(z) * F_{q, i}(z) \quad i \in \mathbb{N} \cup\{0\}=\mathbb{N}_{0},
$$

where:

$$
F_{q, i}(z)=z+\sum_{n=2}^{\infty}[n, q]^{i} z^{n}
$$

using (3) and (4), the power series of $\mathcal{D}_{q}^{i} f(z)$ for $f$ of the form (1) is given by:

$$
\mathcal{D}_{q}^{i} f(z)=z+\sum_{n=2}^{\infty}[n, q]^{i} a_{n} z^{n}
$$

Note that:

$$
\begin{gathered}
\lim _{q \rightarrow 1^{-}} F_{q, i}(z)=z+\sum_{n=2}^{\infty} n^{i} z^{n} . \\
\lim _{q \rightarrow 1^{-}} \mathcal{D}_{q}^{i} f(z)=f(z) *\left(z+\sum_{n=2}^{\infty} n^{i} a_{n} z^{n}\right),
\end{gathered}
$$

which is the familiar Salagean derivative [8].

\section{Methods}

By taking motivation from the above-cited work, we introduce a new subclass $\mathcal{U}_{i, j}(q, \beta, A, B)$. This class is introduced by using the Salagean $q$-differential operator with the concept of Janowski functions. Our defined class generalizes many classes by choosing particular values of the parameters involved in defining this class of functions.

Definition 1. Let $\mathcal{U}_{i, j}(q, \beta, A, B)$ denote the subclass of $\mathcal{A}$ consisting of functions $f$ of the form $(1)$ and satisfy the following subordination condition,

$$
\frac{\mathcal{D}_{q}^{i} f(z)}{\mathcal{D}_{q}^{j} f(z)}-\beta\left|\frac{\mathcal{D}_{q}^{i} f(z)}{\mathcal{D}_{q}^{j} f(z)}-1\right| \prec \frac{1+A z}{1+B z},
$$


where $-1 \leq B<A \leq 1, \beta \geq 0, i \in \mathbb{N}, j \in \mathbb{N}_{0}, i>j, q \in(0,1), z \in E$.

By taking specific values of parameters, we obtain many important subclasses studied by various authors in earlier papers. Here, we enlist some of them.

(i) For $q \rightarrow 1$, and $A=1-2 \alpha, B=-1$, the class $\mathcal{U}_{i, j}(q, \beta, A, B)$ reduces to the class $N_{i, j}(\alpha, \beta),(0 \leq \alpha<1)$ studied by Eker and Owa [33].

(ii) For $q \rightarrow 1, A=1-2 \alpha, B=-1, i=1$, and $j=0$, the class $\mathcal{U}_{i, j}(q, \beta, A, B)$ reduces to the class $\mathcal{U S}(\alpha, \beta)$, $(0 \leq \alpha<1)$ studied by Shams et al. [34].

(iii) For $q \rightarrow 1, A=1-2 \alpha, B=-1, i=2$, and $j=1$, the class $\mathcal{U}_{i, j}(q, \beta, A, B)$ reduces to the class $\mathcal{U K}(\alpha, \beta),(0 \leq \alpha<1)$ studied by Shams et al. [35].

(iv) For $q \rightarrow 1, \beta=0, i=1$, and $j=0$, the class $\mathcal{U}_{i, j}(q, \beta, A, B)$ reduces to the class $\mathcal{S}^{*}(A, B)$, studied by Janowski [36].

(v) For $q \rightarrow 1, \beta=0, i=2$, and $j=1$, the class $\mathcal{U}_{i, j}(q, \beta, A, B)$ reduces to the class $\mathcal{K}(A, B)$, studied by Padmanabhan and Ganesan [37].

Definition 2. Let $\mathcal{T}$ denote the subclass of functions of $\mathcal{A}$ of the form:

$$
f(z)=z-\sum_{n=2}^{\infty} a_{n} z^{n}, \quad a_{n} \geq 0 .
$$

Further, we define the class $\mathcal{T} \mathcal{U}_{i, j}(q, \beta, A, B)=\mathcal{U}_{i, j}(q, \beta, A, B) \cap \mathcal{T}$.

For suitable choices of the parameters $q, \beta, A, B, i$, and $j$, we can get various known or new subclasses of $\mathcal{T}$. For example, we have the following.

(i) $\mathcal{T U}_{j+1, j}(0, \beta, 1-2 \alpha,-1)=\mathcal{T S}(j, \alpha, \beta),\left(0 \leq \alpha<1, \beta \geq 0, j \in N_{0}\right)$ (see Rosy and Murugusundaramoorthy [38] and Aouf [39]).

(ii) $\mathcal{T U}_{1,0}(0,1,1-2 \alpha,-1)=\mathcal{S}_{p} \mathcal{T}(\alpha)$ and $\mathcal{T} \mathcal{U}_{2,1}(0,1,1-2 \alpha,-1)=\mathcal{U C T}(\alpha),(0 \leq \alpha<1)$ (see Bharati et al. [9]).

(iii) $\mathcal{T U}_{1,0}(0,0,1-2 \alpha,-1)=\mathcal{T}^{*}(\alpha)$ and $\mathcal{T U}_{2,1}(0,0,1-2 \alpha,-1)=\mathcal{C}(\alpha),(0 \leq \alpha<1)$ (see Silverman [40]).

\section{Main Results}

In this section, we will prove our main results.

\section{Coefficient estimates:}

Theorem 1. A function $f$ of the form (1) is in the class $\mathcal{U}_{i, j}(q, \beta, A, B)$ if:

$$
\sum_{n=2}^{\infty}\left\{(1+\beta(1+|B|))\left([n, q]^{i}-[n, q]^{j}\right)+\left|B[n, q]^{i}-A[n, q]^{j}\right|\right\} a_{n} \leq A-B .
$$

Proof. It is sufficient to show that:

$$
\left|\frac{p(z)-1}{A-B p(z)}\right|<1 \text {, }
$$

where:

$$
p(z)=\frac{\mathcal{D}_{q}^{i} f(z)}{\mathcal{D}_{q}^{j} f(z)}-\beta\left|\frac{\mathcal{D}_{q}^{i} f(z)}{\mathcal{D}_{q}^{j} f(z)}-1\right| .
$$


We have:

$$
\begin{aligned}
& \left|\frac{p(z)-1}{A-B p(z)}\right| \\
= & \left|\frac{D_{q}^{i} f(z)-D_{q}^{j} f(z)-\beta e^{i \theta}\left|D_{q}^{i} f(z)-D_{q}^{j} f(z)\right|}{A D_{q}^{j} f(z)-B\left[D_{q}^{i} f(z)-\beta e^{i \theta}\left|D_{q}^{i} f(z)-D_{q}^{j} f(z)\right|\right]}\right| \\
= & \left|\frac{\sum_{n=2}^{\infty}\left\{\left([n, q]^{i}-[n, q]^{j}\right) a_{n} z^{n}-\beta e^{i \theta}\left|\sum_{n=2}^{\infty}\left([n, q]^{i}-[n, q]^{j}\right) a_{n} z^{n}\right|\right\}}{(A-B) z-\left[\sum_{n=2}^{\infty}\left(B[n, q]^{i}-A[n, q]^{j}\right) a_{n} z^{n}-B \beta e^{i \theta}\left|\sum_{n=2}^{\infty}\left([n, q]^{i}-[n, q]^{j}\right) a_{n} z^{n}\right|\right]}\right| \\
\leq & \frac{\sum_{n=2}^{\infty}\left([n, q]^{i}-[n, q]^{j}\right)\left|a_{n}\right||z|^{n}+\beta \sum_{n=2}^{\infty}\left([n, q]^{i}-[n, q]^{j}\right)\left|a_{n}\right||z|^{n}}{(A-B)|z|-\left[\sum_{n=2}^{\infty}\left|B[n, q]^{i}-A[n, q]^{j}\right|\left|a_{n}\right||z|^{n}+\beta|B| \sum_{n=2}^{\infty}\left([n, q]^{i}-[n, q]^{j}\right)\left|a_{n}\right||z|^{n}\right]} \\
\leq & \frac{\sum_{n=2}^{\infty}\left([n, q]^{i}-[n, q]^{j}\right)(1+\beta)\left|a_{n}\right|}{(A-B)-\sum_{n=2}^{\infty}\left|B[n, q]^{i}-A[n, q]^{j}\right|\left|a_{n}\right|-\beta|B| \sum_{n=2}^{\infty}\left([n, q]^{i}-[n, q]^{j}\right)\left|a_{n}\right|} .
\end{aligned}
$$

This last expression is bounded above by one if:

$$
\sum_{n=2}^{\infty}\left\{(1+\beta(1+|B|))\left([n, q]^{i}-[n, q]^{j}\right)+\left|B[n, q]^{i}-A[n, q]^{j}\right|\right\} a_{n} \leq A-B
$$

and hence, the proof is completed.

Theorem (2) shows that the condition (7) is also necessary for functions $f$ of the form (6) to be in the class $\mathcal{T} \mathcal{U}_{i, j}(q, \beta, A, B)$.

Theorem 2. Let $f \in \mathcal{T}$. Then, $f \in \mathcal{T} \mathcal{U}_{i, j}(q, \beta, A, B)$ if and only if:

$$
\sum_{n=2}^{\infty}\left\{(1+\beta(1+|B|))\left([n, q]^{i}-[n, q]^{j}\right)+\left|B[n, q]^{i}-[n, q]^{j}\right|\right\} a_{n} \leq A-B .
$$

Proof. Since $\mathcal{T} \mathcal{U}_{i, j}(q, \beta, A, B) \subset \mathcal{U}_{i, j}(q, \beta, A, B)$, for functions $f \in \mathcal{T} \mathcal{U}_{i, j}(q, \beta, A, B)$, we can write:

$$
\left|\frac{p(z)-1}{A-B p(z)}\right|<1
$$

where:

$$
p(z)=\frac{\mathcal{D}_{q}^{i} f(z)}{\mathcal{D}_{q}^{j} f(z)}-\beta\left|\frac{\mathcal{D}_{q}^{i} f(z)}{\mathcal{D}_{q}^{j} f(z)}-1\right|
$$

then:

$$
\begin{aligned}
& \left|\sum_{n=2}^{\infty}\left\{\left([n, q]^{i}-[n, q]^{j}\right) a_{n} z^{n}+\beta e^{i \theta}\left|\sum_{n=2}^{\infty}\left([n, q]^{i}-[n, q]^{j}\right) a_{n} z^{n}\right|\right\}\right| \\
& \quad \times\left\{\begin{array}{c}
(A-B) z+\sum_{n=2}^{\infty}\left(B[n, q]^{i}-A[n, q]^{j}\right) a_{n} z^{n} \\
+B \beta e^{i \theta}\left|\sum_{n=2}^{\infty}\left([n, q]^{i}-[n, q]^{j}\right) a_{n} z^{n}\right|
\end{array}\right\}
\end{aligned} \mid<1 .
$$


Since $\operatorname{Re}(z) \leq|z|$, then we obtain:

$$
\operatorname{Re}\left\{\begin{array}{c}
\sum_{n=2}^{\infty}\left\{\left([n, q]^{i}-[n, q]^{j}\right) a_{n} z^{n}+\beta e^{i \theta}\left|\sum_{n=2}^{\infty}\left([n, q]^{i}-[n, q]^{j}\right) a_{n} z^{n}\right|\right\} \\
\times\left\{\begin{array}{c}
(A-B) z+\sum_{n=2}^{\infty}\left(B[n, q]^{i}-A[n, q]^{j}\right) a_{n} z^{n} \\
+B \beta e^{i \theta}\left|\sum_{n=2}^{\infty}\left([n, q]^{i}-[n, q]^{j}\right) a_{n} z^{n}\right|
\end{array}\right\}
\end{array}\right\}<1 .
$$

Now choosing $z$ to be real and letting $z \rightarrow 1^{-}$, we obtain:

$$
\sum_{n=2}^{\infty}\left\{(1+\beta(1-B))\left([n, q]^{i}-[n, q]^{j}\right)-\left|B[n, q]^{i}-A[n, q]^{j}\right|\right\} a_{n} \leq A-B
$$

or equivalently:

$$
\sum_{n=2}^{\infty}\left\{(1+\beta(1+|B|))\left([n, q]^{i}-[n, q]^{j}\right)+\left|B[n, q]^{i}-A[n, q]^{j}\right|\right\} a_{n} \leq A-B .
$$

This completes the proof.

Corollary 1. Let $f$ be defined by (6) be in the class $\mathcal{T} \mathcal{U}_{i, j}(q, \beta, A, B)$. Then:

$$
a_{n} \leq \frac{A-B}{\left\{(1+\beta(1+|B|))\left([n, q]^{i}-[n, q]^{j}\right)+\left|B[n, q]^{i}-A[n, q]^{j}\right|\right\}}, n \geq 2 .
$$

The result is sharp for the function:

$$
f(z)=z-\frac{A-B}{\left\{(1+\beta(1+|B|))\left([n, q]^{i}-[n, q]^{j}\right)+\left|B[n, q]^{i}-A[n, q]^{j}\right|\right\}} z^{2}, n \geq 2 .
$$

That is, equality can be attained for the function defined in (9).

Distortion theorems:

Theorem 3. Let the function $f$ be defined by (6) be in the class $\mathcal{T} \mathcal{U}_{i, j}(q, \beta, A, B)$. Then:

$$
|f(z)| \geq|z|-\frac{A-B}{\left\{(1+\beta(1+|B|))\left([2, q]^{i}-[2, q]^{j}\right)+\left|B[2, q]^{i}-A[2, q]^{j}\right|\right\}}|z|^{2},
$$

and:

$$
|f(z)| \leq|z|+\frac{A-B}{\left\{(1+\beta(1+|B|))\left([2, q]^{i}-[2, q]^{j}\right)+\left|B[2, q]^{i}-A[2, q]^{j}\right|\right\}}|z|^{2}
$$

The result is sharp.

Proof. In view of Theorem 2, consider the function:

$$
\Phi(n)=\left\{(1+\beta(1+|B|))\left([n, q]^{i}-[n, q]^{j}\right)+\left|B[n, q]^{i}-A[n, q]^{j}\right|\right\} .
$$


Then, it is clear that it is an increasing function of $n(n \geq 2)$; therefore:

$$
\Phi(2) \sum_{n=2}^{\infty}\left|a_{n}\right| \leq \sum_{n=2}^{\infty} \Phi(n)\left|a_{n}\right| \leq A-B .
$$

That is:

$$
\sum_{n=2}^{\infty}\left|a_{n}\right| \leq \frac{A-B}{\Phi(2)}
$$

Thus, we have:

$$
\begin{gathered}
|f(z)| \leq|z|+|z|^{2} \sum_{n=2}^{\infty}\left|a_{n}\right|, \\
|f(z)| \leq|z|+\frac{A-B}{\left\{(1+\beta(1+|B|))\left([2, q]^{i}-[2, q]^{j}\right)+\left|B[2, q]^{i}-A[2, q]^{j}\right|\right\}}|z|^{2} .
\end{gathered}
$$

Similarly, we get:

$$
\begin{aligned}
|f(z)| & \geq|z|-\sum_{n=2}^{\infty}\left|a_{n}\right||z|^{n} \geq|z|-|z|^{2} \sum_{n=2}^{\infty}\left|a_{n}\right| \\
& \geq|z|-\frac{A-B}{\left\{(1+\beta(1+|B|))\left([2, q]^{i}-[2, q]^{j}\right)+\left|B[2, q]^{i}-A[2, q]^{j}\right|\right\}}|z|^{2} .
\end{aligned}
$$

Finally, the equality can be attained for the function:

$$
f(z)=z-\frac{A-B}{\left\{(1+\beta(1+|B|))\left([2, q]^{i}-[2, q]^{j}\right)+\left|B[2, q]^{i}-A[2, q]^{j}\right|\right\}} z^{2} .
$$

At $|z|=r$ and $z=r e^{i(2 k+1) \pi}(k \in \mathbb{Z})$. This completes the result.

Theorem 4. Let the function $f$ be defined by (6) in the class $\mathcal{T} \mathcal{U}_{i, j}(q, \beta, A, B)$. Then:

$$
\left|f^{\prime}(z)\right| \geq 1-\frac{2(A-B)}{\left\{(1+\beta(1+|B|))\left([2, q]^{i}-[2, q]^{j}\right)+\left|B[2, q]^{i}-A[2, q]^{j}\right|\right\}}|z|,
$$

and:

$$
\left|f^{\prime}(z)\right| \leq 1+\frac{2(A-B)}{\left\{(1+\beta(1+|B|))\left([2, q]^{i}-[2, q]^{j}\right)+\left|B[2, q]^{i}-A[2, q]^{j}\right|\right\}}|z| .
$$

The result is sharp.

Proof. As $\frac{\Phi(n)}{n}$ is an increasing function for $n(n \geq 2)$, in view of Theorem 2, we have:

$$
\frac{\Phi(2)}{2} \sum_{n=2}^{\infty} n\left|a_{n}\right| \leq \sum_{n=2}^{\infty} \frac{\Phi(n)}{n} n\left|a_{n}\right|=\sum_{n=2}^{\infty} \Phi(n)\left|a_{n}\right| \leq(A-B)
$$

that is:

$$
\sum_{n=2}^{\infty} n\left|a_{n}\right| \leq \frac{2(A-B)}{\Phi(2)} .
$$

Thus, we have:

$$
\left|f^{\prime}(z)\right| \leq 1+|z| \sum_{n=2}^{\infty} n\left|a_{n}\right|,
$$




$$
|f(z)| \leq 1+\frac{2(A-B)}{\left\{(1+\beta(1+|B|))\left([2, q]^{i}-[2, q]^{j}\right)+\left|B[2, q]^{i}-A[2, q]^{j}\right|\right\}}|z| .
$$

Similarly, we get:

$$
\begin{aligned}
|f(z)| & \geq 1-|z| \sum_{n=2}^{\infty} n\left|a_{n}\right| \\
& \geq 1-\frac{2(A-B)}{\left\{(1+\beta(1+|B|))\left([2, q]^{i}-[2, q]^{j}\right)+\left|B[2, q]^{i}-A[2, q]^{j}\right|\right\}}|z| .
\end{aligned}
$$

Finally, we can see that the assertions of the theorem are sharp for the function $f(z)$ defined by (10). This completes the proof.

Radii of starlikeness, convexity and close-to-convexity:

Theorem 5. Let the function $f$ be defined by (6) be in the class $\mathcal{T} \mathcal{U}_{i, j}(q, \beta, A, B)$. Then:

(i) $f$ is starlike of order $\varphi(0 \leq \varphi<1)$ in $|z|<r_{1}$, where:

$$
r_{1}=\inf _{n \geq 2}\left\{\frac{\left\{(1+\beta(1+|B|))\left([n, q]^{i}-[n, q]^{j}\right)+\left|B[n, q]^{i}-A[n, q]^{j}\right|\right\}}{(A-B)} \times\left(\frac{1-\varphi}{n-\varphi}\right)\right\}^{\frac{1}{n-1}} .
$$

(ii) $f$ is convex of order $\varphi(0 \leq \varphi<1)$ in $|z|<r_{2}$, where:

$$
r_{2}=\inf _{n \geq 2}\left\{\frac{\left\{(1+\beta(1+|B|))\left([n, q]^{i}-[n, q]^{j}\right)+\left|B[n, q]^{i}-A[n, q]^{j}\right|\right\}}{(A-B)} \times\left(\frac{1-\varphi}{n(n-\varphi)}\right)\right\}^{\frac{1}{n-1}} .
$$

(iii) $f$ is close to convex of order $\varphi(0 \leq \varphi<1)$ in $|z|<r_{3}$, where:

$$
r_{3}=\inf _{n \geq 2}\left\{\frac{\left\{(1+\beta(1+|B|))\left([n, q]^{i}-[n, q]^{j}\right)+\left|B[n, q]^{i}-A[n, q]^{j}\right|\right\}}{(A-B)} \times\left(\frac{1-\varphi}{n}\right)\right\}^{\frac{1}{n-1}} .
$$

Each of these results is sharp for the function $f$ given by (9).

Proof. It is sufficient to show that:

$$
\left|\frac{z f^{\prime}(z)}{f(z)}-1\right| \leq 1-\varphi \text { for }|z|<r_{1}
$$

where $r_{1}$ is given by (11). Indeed, we find from (6) that:

$$
\left|\frac{z f^{\prime}(z)}{f(z)}-1\right| \leq \frac{\sum_{n=2}^{\infty}(n-1) a_{n}|z|^{n-1}}{1-\sum_{n=2}^{\infty} a_{n}|z|^{n-1}} .
$$

Thus, we have:

$$
\left|\frac{z f^{\prime}(z)}{f(z)}-1\right| \leq 1-\varphi,
$$

if and only if:

$$
\frac{\sum_{n=2}^{\infty}(n-\varphi) a_{n}|z|^{n-1}}{(1-\varphi)} \leq 1 .
$$


From Theorem 2, the relation (14) is true if:

$$
\left(\frac{n-\varphi}{1-\varphi}\right)|z|^{n-1} \leq \frac{\left\{(1+\beta(1+|B|))\left([n, q]^{i}-[n, q]^{j}\right)+\left|B[n, q]^{i}-A[n, q]^{j}\right|\right\}}{(A-B)}
$$

That is, if:

$$
|z| \leq\left\{\frac{\left\{(1+\beta(1+|B|))\left([n, q]^{i}-[n, q]^{j}\right)+\left|B[n, q]^{i}-A[n, q]^{j}\right|\right\}}{(A-B)} \times\left(\frac{1-\varphi}{n-\varphi}\right)\right\}^{\frac{1}{n-1}}, \text { for } n \geq 2
$$

This implies that:

$$
r_{1}=\inf _{n \geq 2}\left\{\frac{\left\{(1+\beta(1+|B|))\left([n, q]^{i}-[n, q]^{j}\right)+\left|B[n, q]^{i}-A[n, q]^{j}\right|\right\}}{(A-B)} \times\left(\frac{1-\varphi}{n-\varphi}\right)\right\}^{\frac{1}{n-1}}, \text { for } n \geq 2
$$

This completes the proof of (11).

To prove (12) and (13), it is sufficient to show that:

$$
\left|1+\frac{z f^{\prime \prime}(z)}{f^{\prime}(z)}-1\right| \leq 1-\varphi \quad\left(|z|<r_{2}, 0 \leq \varphi<1\right)
$$

and:

$$
\left|f^{\prime}(z)-1\right| \leq 1-\varphi \quad\left(|z|<r_{3}, 0 \leq \varphi<1\right)
$$

respectively.

Extreme points:

Theorem 6. Let

$$
f_{1}(z)=z
$$

and:

$$
f_{n}(z)=z-\frac{(A-B)}{\left\{(1+\beta(1+|B|))\left([n, q]^{i}-[n, q]^{j}\right)+\left|B[n, q]^{i}-A[n, q]^{j}\right|\right\}} z^{n}, n=2,3, \cdots .
$$

Then, $f \in \mathcal{T} \mathcal{U}_{i, j}(q, \beta, A, B)$ if and only if it can be expressed in the following form:

$$
f(z)=\sum_{n=1}^{\infty} \eta_{n} f_{n}(z)
$$

where:

$$
\eta_{n} \geq 0, \quad \sum_{n=1}^{\infty} \eta_{n}=1
$$

Proof. Suppose that:

$$
\begin{aligned}
f(z) & =\sum_{n=1}^{\infty} \eta_{n} f_{n}(z) \\
& =z-\sum_{n=2}^{\infty} \eta_{n} \frac{(A-B)}{\left\{(1+\beta(1+|B|))\left([n, q]^{i}-[n, q]^{j}\right)+\left|B[n, q]^{i}-A[n, q]^{j}\right|\right\}} z^{n} .
\end{aligned}
$$


Then, from Theorem 2, we have:

$$
\begin{aligned}
& \sum_{n=2}^{\infty}\left[\frac{\left\{(1+\beta(1+|B|))\left([n, q]^{i}-[n, q]^{j}\right)+\left|B[n, q]^{i}-A[n, q]^{j}\right|\right\}(A-B)}{\left\{(1+\beta(1+|B|))\left([n, q]^{i}-[n, q]^{j}\right)+\left|B[n, q]^{i}-A[n, q]^{j}\right|\right\}} \eta_{n}\right] \\
= & (A-B) \sum_{n=2}^{\infty} \eta_{n}=(A-B)\left(1-\eta_{1}\right) \leq(A-B) .
\end{aligned}
$$

Thus, in view of Theorem 2, we find that $f \in \mathcal{T U}_{i, j}(q, \beta, A, B)$. Conversely, let us suppose that $f \in \mathcal{T U}_{i, j}(q, \beta, A, B)$, then:

$$
a_{n} \leq \frac{(A-B)}{\left\{(1+\beta(1+|B|))\left([n, q]^{i}-[n, q]^{j}\right)+\left|B[n, q]^{i}-A[n, q]^{j}\right|\right\}} .
$$

By setting:

$$
\eta_{n}=\frac{\left\{(1+\beta(1+|B|))\left([n, q]^{i}-[n, q]^{j}\right)+\left|B[n, q]^{i}-A[n, q]^{j}\right|\right\}}{(A-B)} a_{n}
$$

with:

$$
\eta_{1}=1-\sum_{n=2}^{\infty} \eta_{n}
$$

we have:

$$
f(z)=\sum_{n=1}^{\infty} \eta_{n} f_{n}(z)
$$

This completes the proof.

Corollary 2. The extreme points of the class $\mathcal{T} \mathcal{U}_{i, j}(q, \beta, A, B)$ are given by:

$$
f_{1}(z)=z,
$$

and:

$$
f_{n}(z)=z-\frac{(A-B)}{\left\{(1+\beta(1+|B|))\left([n, q]^{i}-[n, q]^{j}\right)+\left|B[n, q]^{i}-A[n, q]^{j}\right|\right\}} z^{n}, n=2,3, \cdots .
$$

Integral mean inequalities:

Lemma 1. [41] If the functions $f$ and $g$ are analytic in $E$ with:

$$
f(z) \prec g(z),
$$

then for $p>0$ and $z=r e^{i \theta},(0<r<1)$,

$$
\int_{0}^{2 \pi}|f(z)|^{p} d \theta \leq \int_{0}^{2 \pi}|g(z)|^{p} d \theta .
$$

We now make use of Lemma 1 to prove the following result. 
Theorem 7. Suppose that $f \in \mathcal{T U}_{i, j}(q, \beta, A, B), p>0,-1 \leq B<A \leq 1, \beta>0, i \in N, j \in N_{0}, i>j$, and $f_{2}(z)$ is defined by:

$$
f_{2}(z)=z-\frac{A-B}{\left\{(1+\beta(1+|B|))\left([2, q]^{i}-[2, q]^{j}\right)+\left|B[2, q]^{i}-A[2, q]^{j}\right|\right\}} z^{2},
$$

then for $z=r e^{i \theta},(0<r<1)$, we have:

$$
\int_{0}^{2 \pi}|f(z)|^{p} d \theta \leq \int_{0}^{2 \pi}\left|f_{2}(z)\right|^{p} d \theta
$$

Proof. For:

$$
f(z)=z-\sum_{n=2}^{\infty} a_{n} z^{n}, a_{n} \geq 0,
$$

the relation (15) is equivalent to proving that:

$$
\begin{aligned}
& \int_{0}^{2 \pi}\left|1-\sum_{n=2}^{\infty} a_{n} z^{n-1}\right|^{p} d \theta \\
\leq & \int_{0}^{2 \pi}\left|1-\frac{A-B}{\left\{(1+\beta(1+|B|))\left([2, q]^{i}-[2, q]^{j}\right)+\left|B[2, q]^{i}-A[2, q]^{j}\right|\right\}} z\right|^{p} d \theta .
\end{aligned}
$$

By applying Lemma 1, it suffices to show that:

$$
1-\sum_{n=2}^{\infty} a_{n} z^{n-1} \prec 1-\frac{A-B}{\left\{(1+\beta(1+|B|))\left([2, q]^{i}-[2, q]^{j}\right)+\left|B[2, q]^{i}-A[2, q]^{j}\right|\right\}} z .
$$

By setting:

$$
1-\sum_{n=2}^{\infty} a_{n} z^{n-1}=1-\frac{A-B}{\left\{(1+\beta(1+|B|))\left([2, q]^{i}-[2, q]^{j}\right)+\left|B[2, q]^{i}-A[2, q]^{j}\right|\right\}} w(z),
$$

and using (7), we obtain:

$$
\begin{aligned}
|w(z)| & =\left|\sum_{n=2}^{\infty} \frac{\left\{(1+\beta(1+|B|))\left([2, q]^{i}-[2, q]^{j}\right)+\left|B[2, q]^{i}-A[2, q]^{j}\right|\right\}}{A-B} a_{n} z^{n-1}\right| \\
& \leq|z| \sum_{n=2}^{\infty} \frac{\left\{(1+\beta(1+|B|))\left([2, q]^{i}-[2, q]^{j}\right)+\left|B[2, q]^{i}-A[2, q]^{j}\right|\right\}}{A-B} a_{n} \\
& \leq|z| \sum_{n=2}^{\infty} \frac{\left\{(1+\beta(1+|B|))\left([n, q]^{i}-[n, q]^{j}\right)+\left|B[n, q]^{i}-A[n, q]^{j}\right|\right\}}{A-B} a_{n} \\
& \leq|z|<1 .
\end{aligned}
$$

This completes the proof.

\section{Conclusions}

Here, in our present investigation, we have successfully introduced a new subclass of analytic functions in open unit disk $E$. Many properties and characteristics of this newly-defined function class such as coefficient estimates, distortion theorem, radii of star-likeness, convexity, close-to-convexity, 
extreme points, and integral mean inequalities have been studied. We also highlighted a numbers of known consequences, which are already present in the literature.

Author Contributions: M.N. conceived of the main thoughts; S.H., T.M., and S.K. proved the main theorems; M.D. drafted and revised the paper; all authors read and approved the final manuscript.

Funding: This research received no external funding.

Acknowledgments: The work here is supported by UKMGrant No. GUP-2017-064.

Conflicts of Interest: The authors declare no conflict of interest.

\section{References}

1. Goodman, A.W. Univalent Functions; Polygonal Publishing House: Washington, NJ, USA, 1983; Volumes I and II.

2. Roberston, M.S. On the theory of univalent functions. Ann. Math. 1936, 37, 374-408.

3. Goodman, A.W. On uniformly convex functions. Ann. Polon. Math. 1991, 56, 87-92. [CrossRef]

4. Ma, W.; Minda, D. Uniformly convex functions. Ann. Polon. Math. 1992, 57, 165-175. [CrossRef]

5. Rønning, F. Uniformly convex functions and a corresponding class of starlike functions. Proc. Am. Math. Soc. 1993, 118, 189-196. [CrossRef]

6. Kanas, S.; Wisniowska, A. Conic regions and k-uniform convexity. J. Comput. Appl. Math. 1999, 105, 327-336. [CrossRef]

7. Kanas, S.; Wisniowska, A. Conic domains and k-starlike functions. Rev. Roum. Math. Pure Appl. 2000, 45, 647-657.

8. Salagean, G.S. Subclasses of univalent functions. In Lecture Notes in Mathematics, 1013, Proceedings of the Complex Analysis, Fifth Romanian—Finnish Seminar, Bucharest, Romania, June 28-July 3, 1981; Springer: Berlin, Germany, 1983; pp. 362-372.

9. Bharati, R.; Parvatham, R.; Swaminathan, A. On subclasses of uniformly convex functions and corresponding class of starlike functions. Tamkang J. Math. 1997, 28, 17-32.

10. Aiena, P.; Triolo, S. Local spectral theory for Drazin invertible operators. J. Math. Anal. Appl. 2016, 435, 414-424. [CrossRef]

11. Aldweby, H.; Darus, M. On Fekete-Szegö problems for certain subclasses fefined by $q$-derivative. J. Func. Spac. 2017, 2017, 7156738.

12. Aldweby, H.; Darus, M. Some subordination results on q-analogue of Ruscheweyh differential operator. Abstr. Appl. Anal. 2014, 2014, 958563. [CrossRef]

13. Darus, M.; Hussain, S.; Raza, M.; Sokol, J. On a subclass of starlike functions. Results Math. 2018, 73, 22. [CrossRef]

14. Elhaddad, S.; Darus, M.; Aldweby, H. On certain subclasses of analytic functions involving differential operator. Jnanabha 2018, 48, 53-62.

15. Gangadharan, A.; Shanmugam, T.N.; Srivastava, H.M. Generalized hypergeometric functions associated with k-uniformly convex Functions. Comput. Math. Appl. 2002, 44, 1515-1526. [CrossRef]

16. Kanas, S. Techniques of the differential subordination for domain bounded by conic sections. Int. J. Math. Sci. 2003, 38, 2389-2400. [CrossRef]

17. Hussain, S.; Khan, S.; Zaighum, M.A.; Darus, M. Applications of a q-Salagean type operator on multivalent functions. J. Ineq. Appl. 2018, 2018, 301. [CrossRef]

18. Swaminathan, A. Hypergeometric functions in the parabolic domain. Tamsui Oxf. J. Math. Sci. 2004, 20,1-16.

19. Miller, S.S.; Mocanu, P.T. Differential Subordinations, Theory and Applications. In Monographs and Textbooks in Pure and Application Mathematics; Marcel Dekker Inc.: New York, NY, USA; Basel, Switzerland, 2000; Volume 225.

20. Carmichael, R.D. The general theory of linear q-difference equations. Am. J. Math. 1912, 34, 147-168. [CrossRef]

21. Jackson, F.H. On q-definite integrals. Quart. J. Pure Appl. Math. 1910, 41, 193-203.

22. Mason, T.E. On properties of the solution of linear q-difference equations with entire function coefficients. Am. J. Math. 1915, 37, 439-444. [CrossRef]

23. Trjitzinsky, W.J. Analytic theory of linear q-difference equations. Acta Math. 1933, 61, 1-38. [CrossRef] 
24. Ismail, M.E.H.; Merkes, E.; Styer, D. A generalization of starlike functions. Complex Var. Theory Appl. 1990, 14, 77-84. [CrossRef]

25. Bongiorno, B.; Trapani, C.; Triolo, S. Extensions of positive linear functionals on a topological *-algebra. Rocky Mt. J. Math. 2010, 40, 1745-1777. [CrossRef]

26. Fernandez, A.; Baleanu, D.; Srivastava, H.M. Series representations for fractional-calculus operators involving generalised Mittag-Leffler functions. Commun. Nonlinear Sci. Numer. Simul. 2019, 67, 517-527. [CrossRef]

27. Shareef, Z.; Hussain, S.; Darus, M. Convolution operator in geometric functions Theory. J. Ineq. Appl. 2012, 2012, 213. [CrossRef]

28. Srivastava, H.M.; Juma, A.R.S.; Zayed, H.M. Univalence conditions for an integral operator defined by a generalization of the Srivastava-Attiya operator. Filomat 2018, 32, 2101-2114. [CrossRef]

29. Srivastava, H.M.; Günerhan, H. Analytical and approximate solutions of fractional-order susceptible-infected -recovered epidemic model of childhood disease. Math. Methods Appl. Sci. 2019, 42, 935-941. [CrossRef]

30. Trapani, C.; Triolo, S. Representations of modules over a *-algebra and related seminorms. Studia Math. 2008, 184, 133-148. [CrossRef]

31. Andrews, G.E.; Askey, G.E.; Roy, R. Special Functions; Cambridge University Press: Cambridge, UK, 1999.

32. Govindaraj, M.; Sivasubramanian, S. On a class of analytic functions related to conic domains involving q-calculus. Anal. Math. 2017, 43, 475-487. [CrossRef]

33. Eker, S.S.; Owa, S. Certain classes of analytic functions involving Salagean operator. J. Ineq. Pure Appl. Math. 2009, 10, 12-22.

34. Shams, S.; Kulkarni, S.R.; Jahangiri, J.M. On a class of univalent functions defined by Ruschweyh derivatives. Kyungpook Math. J. 2003, 43, 579-585.

35. Shams, S.; Kulkarni, S.R.; Jahangiri, J.M. Classes of uniformly starlike and convex functions. Int. J. Math. Math. Sci. 2004, 53, 2959-2961. [CrossRef]

36. Janowski, W. Some extremal problems for certain families of analytic functions. Ann. Polon. Math. 1973, 28, 648-658. [CrossRef]

37. Padmanabhan, K.S.; Ganesan, M.S. Convolutions of certain classes of univalent functions with negative coefficients. Indian J. Pure Appl. Math. 1988, 19, 880-889.

38. Rosy, T.; Murugusundaramoorthy, G. Fractional calculus and their applications to certain subclass of uniformly convex functions. Far East J. Math. Sci. 2004, 15, 231-242.

39. Aouf, M.K. A subclass of uniformly convex functions with negative coefficients. Mathematica 2010, 52, 99-111.

40. Silverman, H. Univalent functions with negative coefficients. Proc. Am. Math. Soc. 1975, 51, $109-116$. [CrossRef]

41. Littlewood, J.E. On inequalities in the theory of functions. Proc. Lond. Math. Soc. 1925, 23, 481-519. [CrossRef] 